\title{
Altered electrophysiological properties and deranged cardiac autonomic modulation predispose patients to atrial fibrillation after arrested heart operations
}

\author{
JM Kalisnik ${ }^{* *}$, A Hrastovec ${ }^{1}$, V Avbelj², B Gersak ${ }^{1}$ \\ From 23rd World Congress of the World Society of Cardio-Thoracic Surgeons \\ Split, Croatia. 12-15 September 2013
}

\section{Background}

Advanced Heart Rate (HR) Variability analyses applying non-linear dynamics and chaos theory provide information about derangement of cardiac autonomic modulation predicting ventricular or atrial arrhythmias. Aim of the present study was to determine which high-resolution ECG and non-linear HR dynamics parameters predispose to development of postoperative atrial fibrillation after surgery on the open arrested heart.

\section{Methods}

43 consecutive patients, 26 men, mean age $70.3 \mathrm{yrs}$ referred either for isolated aortic valve replacement \pm concomitant coronary revascularization or Bentall procedure were enrolled into the study. High-resolution 20-minute ECG recordings were performed one day before operation to determine RR, PQ, QT and QTc interval as well as non-linear HR parameters by Detrended Fluctuation Analysis (DFA) with short-( $\leq 11$ beats) and long-term (> 11 beats) correlation properties of R-R intervals. Statistical analyses included paired-samples t-test, Mann-Whitney or Fischer exact test. Results were reported as mean $\pm \mathrm{SE}$; $\mathrm{p}<0.05$ or less was considered significant.

\section{Results}

Out of 43 patients 26 developed AF after operation (AF group) and 17 did not (noAF group). The two groups had similar demographic and perioperative characteristics. Cardiopulmonary bypass time $(112 \pm 28$ vs. $97 \pm 30$

\footnotetext{
* Correspondence: jmkalisnik@gmail.com

'Department of Cardiovascular Surgery, University Clinical Center Ljubljana, Slovenia

Full list of author information is available at the end of the article
}

min; $\mathrm{p}=0.11)$ and aortic cross-clamp time ( $83 \pm 22$ vs. $76 \pm 27$ min; $\mathrm{p}=0.15$ ) tended to be longer in AF group. There were no differences in RR, QT or QTc interval between AF and noAF group (64 \pm 11 vs. $65 \pm 10,420$ \pm 32 vs. $436 \pm 51$ and $432 \pm 26$ vs. $452 \pm 55 \mathrm{~ms}$, respectively; $\mathrm{p}=\mathrm{NS}$ ). DFA parameter $\alpha_{1}$ tended to be higher and DFA $\alpha_{2}$ proved consistently higher in AF group $(0.98 \pm 0.36$ vs. $0.86 \pm 0.28 ; \mathrm{p}=0.26$ and $0.89 \pm 0.17$ vs. $0.76 \pm 0.18 ; \mathrm{p}=0.018)$. In addition, $\mathrm{PQ}$ interval was consistently shorter $(160 \pm 20 v s .184 \pm 44 ; \mathrm{p}=0.033)$ in AF group.

\section{Conclusions}

We describe for the first time that patients prone to postoperative AF after arrested heart surgery exhibit profoundly altered non-linear Heart Rate dynamics and shorter PQ interval already preoperatively and independently of perioperative factors. In accordance with the results from our previous beating heart studies, parameter DFA $\alpha_{2}$ comprehensively indicates higher risk of postoperative atrial fibrillation occurence.

\section{Authors' details \\ 'Department of Cardiovascular Surgery, University Clinical Center Ljubljana, Slovenia. ${ }^{2}$ Department of Communications and Computer Networks, Jozef Stefan Institute, Ljubljana, Slovenia.}

Published: 11 September 2013

\section{doi:10.1186/1749-8090-8-S1-062}

Cite this article as: Kalisnik et al:: Altered electrophysiological properties and deranged cardiac autonomic modulation predispose patients to atrial fibrillation after arrested heart operations. Journal of Cardiothoracic Surgery 2013 8(Suppl 1):O62.

\section{Biomed Central}

C 2013 Kalisnik et al; licensee BioMed Central Ltd. This is an Open Access article distributed under the terms of the Creative Commons Attribution License (http://creativecommons.org/licenses/by/2.0), which permits unrestricted use, distribution, and reproduction in any medium, provided the original work is properly cited. 